\title{
Chiral Recognition of Organic Molecules by Atomic Kinks on Surfaces
}

\author{
T. Greber, ${ }^{1}$ Ž. Šljivančanin, ${ }^{2}$ R. Schillinger, ${ }^{1,3}$ J. Wider, ${ }^{1,3, *}$ and B. Hammer ${ }^{4}$ \\ ${ }^{1}$ Physik Institut, Universität Zürich, Winterthurerstrasse 190, CH-8057 Zürich, Switzerland \\ ${ }^{2}$ École Polytechnique Fédérale de Lausanne (EPFL), Institut Romand de Recherche Numérique en Physique des Matériaux (IRRMA), \\ CH-1015 Lausanne, Switzerland \\ ${ }^{3}$ Paul Scherrer Institut, CH-5232 Villigen, Switzerland \\ ${ }^{4}$ Department of Physics and Astronomy and iNANO, University of Aarhus, Denmark
}

(Received 12 September 2005; published 9 February 2006)

\begin{abstract}
Two distinct non-mirror-symmetric conformations of $D$ - and $L$-cysteine were found after adsorption on $\mathrm{Au}(17119)^{S}$. This demonstrates chiral heterorecognition, i.e., enantioselectivity of $S$ kinks on vicinal $\mathrm{Au}(111)$. The structures as determined by angle scanned $\mathrm{x}$-ray photoelectron diffraction agree well with those from density functional theory calculations. The calculations predict adsorption energies of $\approx 2 \mathrm{eV}$ where $D$-cysteine binds $140 \mathrm{meV}$ stronger than $L$-cysteine. The classical three point contact model for molecular recognition fails to explain these findings.
\end{abstract}

DOI: $10.1103 /$ PhysRevLett.96.056103

Molecular recognition is among the most fundamental processes in nature. In the lock and key picture a receptor molecule discriminates a target molecule from all other molecules. Beyond this lucent model, the recognition process becomes complex. It involves trajectories on high dimensional potential energy surfaces, is driven by the formation of bonds, comprises energy dissipation and conformational changes of the receptor and the target molecule. The recognition of enantiomers, as it is required for the separation of, e.g., the amino acid $D$-cysteine from $L$-cysteine, requires chiral heterorecognition. This is pivotal for the understanding of life and will, if it can be controlled in heterogeneous catalysis, revolutionize pharmaceutical industries.

For chiral recognition, both the receptor and the target molecules have to be chiral ( $D$ or $L$ ). It requires the formation of receptor-target pairs $(D D, D L, L D, L L)$, where the energy of pure pairs $(D D$ and $L L)$ must be different to that of mixed pairs $(D L$ and $L D)$ [1]. In the case of chiral homorecognition the receptor and the target molecules are identical or enantiomorph [2]. If left $(L)$ and right $(D)$ shall be separated, homorecognition is not sufficient since receptor and target molecules have identical physical properties. Separation of enantiomers requires chiral heterorecognition, i.e., a receptor which is physically different from the target, ideally in two distinct phases. On surfaces it involves an enantiospecific adsorption energy and may be realized via preformation of a chiral structure after adsorption of a nonracemic mixture of molecules [36], or as shown here, by the use of an inorganic, intrinsically chiral surface. After first attempts on chiral silver surfaces, where McFadden et al. found no enantiospecific adsorption energies [7], Sholl made theoretical predictions of enantiomeric shifts in the binding energies of hydrocarbons on platinum [8]. The first experimental observation that naturally chiral metal surfaces are enantiospecific came from Attard et al. [9]. Near room temperature they found for the electro-oxidation of glucose on $\mathrm{Pt}$, enantio-
PACS numbers: 68.43.Fg, 61.14.Qp, 68.43.Bc, 87.15.By

selective effects in the order of $13 \mathrm{meV}(0.3 \mathrm{kcal} / \mathrm{mol})$. Later, Gellman et al. showed, more directly, differences in adsorption energy by thermal desorption spectroscopy (TDS) [10]. For R-3-methylcyclohexanone on $\mathrm{Cu}(643)^{S}$ and $\mathrm{Cu}(634)^{R}$, respectively, they found a shift of $3.5 \mathrm{~K}$ of the desorption peak, which corresponds to an adsorption energy difference of $10 \pm 3 \mathrm{meV}$. Such energy differences indicate chiral heterorecognition on the molecular level. However, for the full understanding of such recognition processes the structure of the adsorbed enantiomers has to be known. This has been realized for chiral homorecognition of cysteine by scanning tunneling microscopy [2] and for the absolute determination of the chirality of tartaric acid on $\mathrm{Cu}(110)$ by x-ray photoelectron diffraction [6]. In both cases, no enantioselectivity, i.e., difference in the adsorption energy between the left- and the right-handed molecules is expected since the experiments were performed on nonchiral face-centered-cubic (110) surfaces. Here, we demonstrate that $L$ - and $D$-cysteine adsorb in two distinct and non-mirror-symmetric conformations on $\mathrm{Au}(17119)^{S}$. From this enantioselectivity may be safely concluded. The structures as determined from density functional theory calculations compare well with the experiment and show an adsorption energy difference between the two enantiomers of $140 \mathrm{meV}$, larger than the value predicted for molecules where the carboxyl group is substituted with a phosphino group (APPT) [11]. Furthermore, the knowledge of the structure allows tests of models for the recognition process. For the present case it turns out that the three point contact model for chiral recognition [12] does not hold.

The experiments have been performed in a customized end station for recording $2 \pi$ x-ray photoelectron diffraction (XPD) at the Swiss Light Source. The diffraction patterns have been measured at room temperature with $768 \mathrm{eV}$ linearly polarized light. The $\mathrm{Au}(17119)$ sample (MaTecK) was cleaned by standard sputtering-annealing cycles and characterized with $\mathrm{x}$-ray photoelectron spec- 
troscopy (XPS), low energy electron diffraction (LEED) and XPD. In Fig. 1 the Au(17 119) substrate is shown. The surface is vicinal to $\mathrm{Au}(111)$ with a miscut angle of $15.4^{\circ}$. The unit cell contains one kink with low coordinated atoms. Such a kink is chiral and not superimposable to its mirror image. It contains 4 atoms, i.e., the kink atom with a coordination $c$ of 6 neighbor metal atoms, the inner kink atom $(c=8)$, a nearby step atom $(c=7)$ and the inner corner atom $(c=11)$. According to Attard's notation $\mathrm{Au}(17119)$ is left handed, i.e., of $S$ type [13]. $D$-cysteine (Sigma, 98\%) and $L$-cysteine (Fluka, $>99 \%)\left(\mathrm{C}_{3} \mathrm{H}_{7} \mathrm{NO}_{2} \mathrm{~S}\right.$ ) have been evaporated from Knudsen cells kept at $400 \mathrm{~K}$, which resulted in deposition rates of about one $\mathrm{ML} / 40 \mathrm{~min}$. The experimental results indicate no significant racemization of the two enantiomers. After deposition of $0.8 \pm 0.1 \mathrm{ML}$ cysteine at room temperature the sample was annealed to $\approx 400 \mathrm{~K}$. One ML corresponds to a coverage of one molecule per $\mathrm{Au}(17119)$ unit cell, or $1.08 \times$ $10^{18}$ molecules $\mathrm{m}^{-2}$. The kinks are so dilute that no strong molecule-molecule interaction is expected, if the molecules bind to the kinks. With XPS cysteine was identified to be in the $\mathrm{SC}_{\beta} \mathrm{H}_{2}-\mathrm{C}_{\alpha} \mathrm{H}-\mathrm{NH}_{2}-\mathrm{COOH}$ state. The $\mathrm{S} 2 p$ binding energy of $162 \mathrm{eV}$ indicates the deprotonation of the sulfur atom, which then strongly binds to the gold substrate [14]. After annealing the $\mathrm{N} 1 s$ binding energy of $399.7 \pm 0.2 \mathrm{eV}$ coincides with that of $\mathrm{NH}_{2}$ and dominates the $\mathrm{NH}_{3}^{+}$species at $1.7 \mathrm{eV}$ higher binding energy. The $\mathrm{COOH}$ group is evidenced by two $\mathrm{O} 1 s$ oxygen species at 531 and $533 \mathrm{eV}$ binding energy.

The density functional theory (DFT) calculations are performed with the DACAPO computer program [15] using the repeated slab approach [16] and the ultrasoft pseudopotential approximation $[17,18]$ with a plane wave basis set for the Kohn-Sham one-electron wave functions. It is the same approach as it was used in a previous publication on enantiospecific adsorption of APPT on Au(17 119) [11]. Absolute adsorption energies calculated with the present method are believed to be converged to the $250 \mathrm{meV}$ level [15] - the use of a finite number of $\mathrm{Au}(111)$ layers in the slab, e.g., causes errors in the $100 \mathrm{meV}$ range [19]. However, relative energies are typically more reliable than absolute energies.

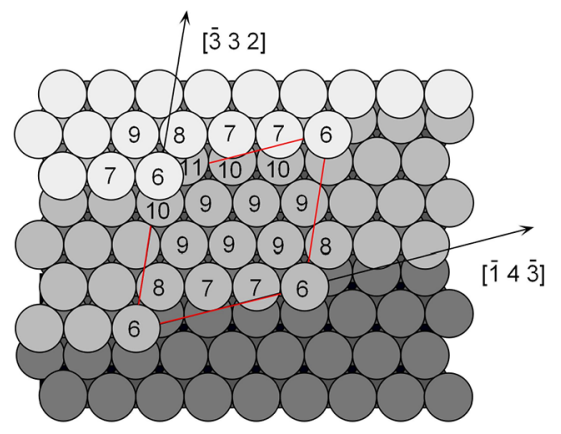

FIG. 1 (color online). Unit cell of the $\mathrm{Au}(17119)$. Coordination numbers are marked. The kink atom $(c=6)$ has lowest coordination.
Figure 2 demonstrates chiral recognition of $D$ - and $L$-cysteine and $\mathrm{Au}\left(\begin{array}{llll}17 & 11 & 9\end{array}\right)^{S}$. The data show the angular dependence of the photoelectron intensities as seen from the emitter. The intensities $I(\theta, \phi)$ are stereographically projected and displayed on a linear gray scale with white to be the highest intensity. Figure 2(b) shows the secondary electron diffraction pattern at $E_{\text {kin }}=486 \mathrm{eV}$. The intensity depressions along the $\langle 110\rangle$ directions indicate the crystal orientation [20]. The pattern has no mirror symmetry and thus indicates chirality of the sample. Figs. 2(c) and 2(d) display the $\mathrm{N} 1 s$ emission patterns at $E_{\text {kin }}=368 \mathrm{eV}$ for $D$ - and $L$-cysteine, respectively. One single forward scattering peak $X_{D}$ or $X_{L}$ dominates both patterns. This signals for $D$ - and $L$-cysteine a single and distinct adsorption geometry. Together with the non-mirror-symmetry of the substrate it is evidence for chiral heterorecognition, i.e., structures with different energy of $D$ - and $L$-cysteine on $\mathrm{Au}(17119)^{S}$.

The $\mathrm{N} 1 s$ forward scattering peaks point along the axis $\mathrm{N}-X$, where $X$ is a neighbor atom which lies above $(\theta<$ $90^{\circ}$ ) the $\mathrm{N}$ emitter. A priori the identity of $X$ is not known. Scattering from hydrogen is too weak to produce this feature. From the diffraction fringes on cones along the $\mathrm{N}-X$ axis the emitter scatterer distance is estimated to be $<2.5 \AA$ A. Comparison with the bond lengths in cysteine therefore suggests this scatterer to be the central carbon atom $\mathrm{C}_{\alpha}$, which gives the absolute orientation of cysteine on $\mathrm{Au}(17119)$.

In Fig. 3 the structure as determined from the experiment is compared with that of the DFT calculations. For a)

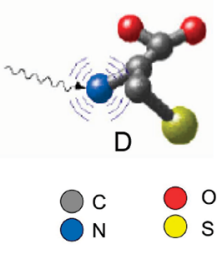

c)

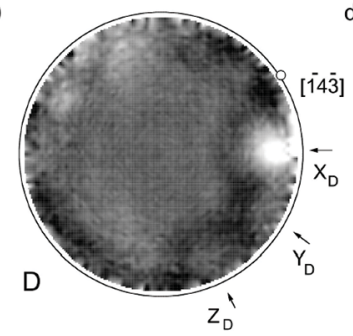

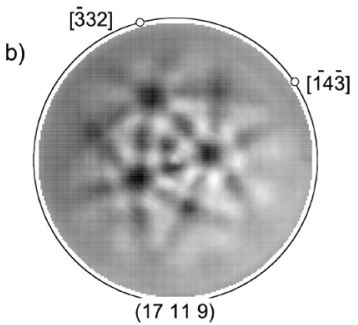

d)

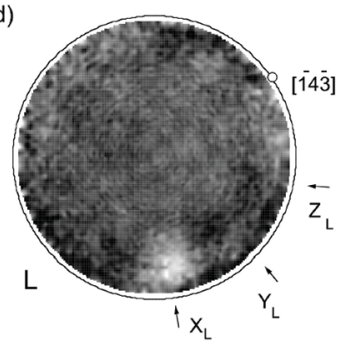

FIG. 2 (color). Angle scanned x-ray photoelectron diffraction patterns. (a) Sketch of a $D$-cysteine molecule without the hydrogen atoms. (b) Secondary electron diffraction pattern $\left(E_{\mathrm{kin}}=\right.$ $486 \mathrm{eV}$ ) from where the orientation and the chirality of the substrate is inferred. (c) $\mathrm{N} 1 s$ emission $\left(E_{\text {kin }}=368 \mathrm{eV}\right)$ for $D$-cysteine and (d) $L$-cysteine. The single forward scattering peaks in (c) and (d) indicate distinct single orientations for the two enantiomers. 
a)
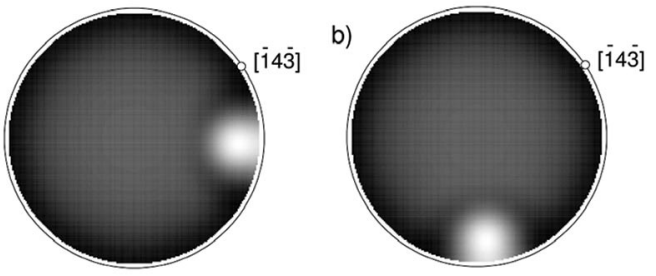

c)
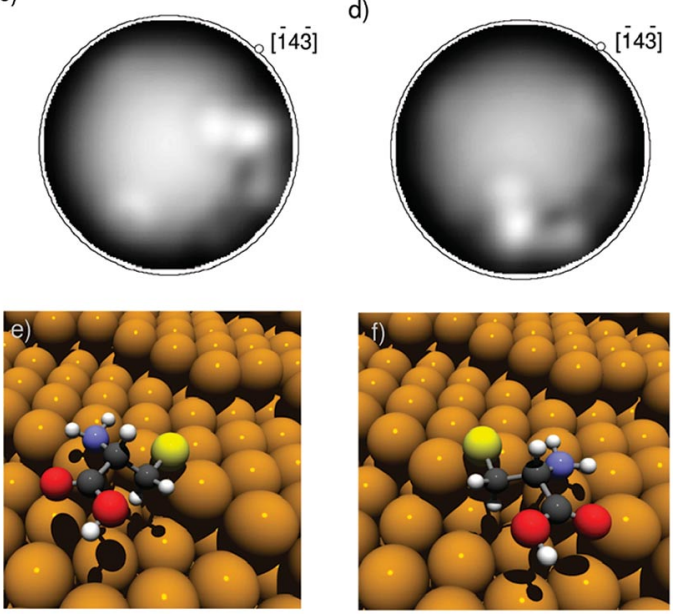

FIG. 3 (color). (a),(b) Single scattering cluster calculations fitting a $\mathrm{N}-\mathrm{C}_{\alpha}$ atomic pair to the experimental data in Fig. 2. (c),(d) Single scattering cluster calculations for the DFT conformation of $D$ - and $L$-cysteine on $\mathrm{Au}(17119)$. (e),(f) Threedimensional images of the adsorption structures of $D$ - and $L$-cysteine.

this purpose the XPD patterns and the equilibrium coordinates from DFT are interpreted with single scattering cluster (SSC) calculations [21]. Figs. 3(a) and 3(b) are the best $\mathrm{N}-\mathrm{C}_{\alpha}$ atomic pair fits to the experimental data in Figs. 2(c) and 2(d). They show SSC calculations for $\mathrm{N}^{-C_{\alpha}}$ pairs with the known interatomic distance of $1.5 \AA$. From this the polar and azimuthal angles of the $\mathrm{N}-\mathrm{C}_{\alpha}$ axis are found (see Table I). The $\mathrm{N}-\mathrm{C}_{\alpha}$ pairs describe the XPD patterns quite well. All other features are weaker and are related to scattering with other atoms in the cysteine molecules. Figs. 3(c) and 3(d) display N 1s SSC patterns as calculated for the structure of $D$ - and $L$-cysteine on $\mathrm{Au}(17119)$ from DFT. Comparison with Figs. 2(c) and 2(d) shows excellent agreement, also with fine features apart from the dominant $\mathrm{N}-\mathrm{C}_{\alpha}$ forward scattering peak and confirm the correspondence between XPD and DFT. The two additional features at grazing angles, $Y$ and $Z$, near the $\mathrm{N}-\mathrm{C}_{\alpha}$ forward scattering peak are well resolved by the experiment in Fig. 2(c) and are due to scattering from the $\mathrm{COOH}$ carboxyl group. In Table I the main structural parameters as derived from the clusters used to simulate Figs. 3(a)-3(d) are summarized. The $\mathrm{N}-\mathrm{C}_{\alpha}$ directions agree better than $\pm 10^{\circ}$ for XPD and DFT. The small deviations may have different origins: The errors due to an inadequate description of the electron scattering process should not be larger than $3^{\circ}$. More importantly, DFT predicts the structure at zero temperature, while the experiments were conducted at room temperature. Taking into account that the lowest molecular torsion modes of cysteine are in the order of $5 \mathrm{meV}$ [22] it may be expected that nonharmonic effects in the, compared to $k_{B} T=25 \mathrm{meV}$, flat total energy surface are present and cause a temperature dependence of the equilibrium conformation. Table I also shows that the elastic deformation energies of the molecules and the substrate have the same order of magnitude as the adsorption energy difference between $D$ - and $L$-cysteine, which is $140 \mathrm{meV}$ [23].

Figs. 3(e) and 3(f) display hard sphere models of the theoretically determined adsorption structures of $D$ - and $L$-cysteine on $\mathrm{Au}(17119)$. Together with the bond energies in Table I they indicate that cysteine forms a thiolate and an amino bond with the gold surface, where the carboxyl group "flies" above the gold substrate. The trend that molecules bind to the lowest coordinated metal atoms $[19,24]$ is nicely reproduced. Both enantiomers bind to the

TABLE I. Orientation of the $\mathrm{N}-\mathrm{C}_{\alpha}$ bond and calculated energies of $D$ - and $L$-cysteine on $\mathrm{Au}(17119)$. The polar angle $\theta$ is measured from the surface normal, the azimuthal angle $\phi$ is measured clockwise from the $[\overline{1} 4 \overline{3}]$ direction. The binding energies of the $\mathrm{S}$, $\mathrm{NH}_{2}$ and $\mathrm{COOH}$ functional groups $E(\mathrm{~S}), E\left(\mathrm{NH}_{2}\right)$, and $E(\mathrm{COOH})$, together with the molecule and the surface deformation energies $-E_{\mathrm{def}}$ (molecule) and $-E_{\mathrm{def}}$ (surface), are calculated as described in Ref. [11], where $1.55 \mathrm{eV}$ have been added to the $\mathrm{S}$ bond in order to account for the thiolate, i.e., the deprotonation of the thiol group upon adsorption. The model binding energy $E_{\text {model }}$ is the sum of the latter five terms.

\begin{tabular}{llcrr}
\hline \hline & & $\begin{array}{c}D \text {-cysteine } \\
\text { Experiment }\end{array}$ & Theory & $\begin{array}{c}\text { L-cysteine } \\
\text { Experiment }\end{array}$ \\
\hline $\mathrm{N}-\mathrm{C}_{\alpha}$ & $\theta$ (deg.) & 74 & 74 & 76 \\
& $\phi$ (deg.) & 35 & 37 & 116 \\
Energy $(\mathrm{eV})$ & $E_{\text {model }}$ & & 2.17 & 126 \\
& $E(\mathrm{~S})$ & & 1.95 & 2.03 \\
& $E\left(\mathrm{NH}_{2}\right)$ & & 0.44 & 1.92 \\
& $E(\mathrm{COOH})$ & -0.05 & 0.25 \\
& $-E_{\text {def }}($ molecule $)$ & -0.07 & -0.03 \\
& $-E_{\text {def }}($ surface $)$ & -0.10 & -0.05 \\
& & & -0.06 \\
\hline \hline
\end{tabular}


kink atom $(c=6)$. For $D$-cysteine it is the amino group, but for $L$-cysteine it is the thiol group. The amino bond to the kink atom $\left(c_{N}^{D}=6\right)$ is indeed stronger than to the step $\left(c_{N}^{L}=7\right)$ atom. On the other hand the thiolate binds, as on $\mathrm{Au}(111)$ [25], to bridge sites, i.e., is shared by two gold atoms. Though, the two bridge sites are not the same for the two enantiomers. The thiol group in $D$-cysteine binds to two step atoms $\left(c_{S}^{D}=7 ; 7\right)$, but that in $L$-cysteine to the kink and the inner kink atom $\left(c_{S}^{L}=6 ; 8\right)$, where the same total coordination of 14 gives an intuitive understanding for the very similar thiolate-bond strength. The third bond, i.e., that of the carboxyl group, is not significant or even weakly repulsive. $L$-cysteine binds not with sulfur nor with nitrogen to the same receptor site and the amino and the thiolate bond form hinges which point in opposite directions $\left(156^{\circ}\right)$.

Enantioselectivity, despite lack of a third bond, a different amino-bond strength, and bonding of specific molecular groups to different receptor sites, is a contradiction to the three point contact model for chiral recognition [12]. In this model the different bonding of enantiomers to a given receptor involves three different, nonequivalent binding sites. Discrimination occurs when one enantiomer interacts with all of the sites, while its enantiomorph binds only at one. Even if we concede that the carboxylic group has to lie parallel to the surface in order to benefit from the surface potential, which translates in a third "quasibond," we have to step beyond a rigid lock and key concept in order to understand why gold kinks are enantioselective to cysteine [26]. This is also reflected in the different deformation energies of the molecules and the substrate (see Table I). We take our findings as an indication that enantiospecific steric repulsion and deformation of receptor and target molecule leads to the situation that $L$-cysteine does not bind in the same way to $S$ kinks as does $D$-cysteine.

In conclusion, enantioselectivity of a chiral gold surface is verified by the experimental determination of two distinct adsorption geometries for $D$ - and $L$-cysteine on $\mathrm{Au}(17119)^{S}$. The results demonstrate chiral heterorecognition of an organic molecule and an inorganic surface. They are supported by density functional theory calculations, which confirm the experimental structures and predict $D$-cysteine to bind $140 \mathrm{meV}$ stronger to $S$ kinks on gold surfaces than it does $L$-cysteine. The molecular equilibrium conformations after adsorption indicate that the gold kinks discriminate enantiomers, where two different bond pairs and enantiospecific deformations were found.

The experiments were performed at the Swiss Light Source, Paul Scherrer Institut, Villigen. Fruitful discussions with Georges Wagnière, Neville Richardson, KarlHeinz Ernst, Jürg Osterwalder, and funding from the Schweizerischen Nationalfonds are gratefully acknowledged. The calculations were performed at the central computational facilities of the École Polytechnique Fédérale de Lausanne and of the Swiss Center for Scientific Computing.
*Present address: Hahn-Meitner-Institut, Glienicker Strasse 100, D-14109 Berlin, Germany.

[1] As long as the weak interaction is neglected, i.e., parity is conserved, chiral recognition does not produce a lifting of degeneracy between left and right. For the energies of the receptor-target pairs $E(D D)=E(L L)$ and $E(D L)=$ $E(L D)$ hold. See, e.g., R. A. Hegstrom, D. W. Rein, and P. G. H. Sandars, J. Chem. Phys. 73, 2329 (1980).

[2] A. Kühnle, T. R. Linderoth, B. Hammer, and F. Besenbacher, Nature (London) 415, 891 (2002).

[3] S. M. Barlow and R. Raval, Surf. Sci. Rep. 50, 201 (2003).

[4] Q. Chen and N. V. Richardson, Nat. Mater. 2, 324 (2003).

[5] A. Kühnle, L. M. Molina, T. R. Linderoth, B. Hammer, and F. Besenbacher, Phys. Rev. Lett. 93, 086101 (2004).

[6] R. Fasel, J. Wider, C. Quitmann, K.-H. Ernst, and T. Greber, Angew. Chem., Int. Ed. Engl. 43, 2853 (2004).

[7] C.F. McFadden, P.S. Cremer, and A. J. Gellman, Langmuir 12, 2483 (1996).

[8] D. S. Sholl, Langmuir 14, 862 (1998).

[9] G. A. Attard, A. Ahmadi, J. Feliu, A. Rodes, E. Herrero, S. Blais, and G. Jerkiewicz, J. Phys. Chem. B 103, 1381 (1999).

[10] A. J. Gellman, J. D. Horvath, and M. T. Buelow, J. Mol. Catal. A: Chem. 167, 3 (2001).

[11] Ž. Šljivančanin, K. V. Gothelf, and B. Hammer, J. Am. Chem. Soc. 124, 14789 (2002).

[12] L.H. Easson and E. Stedman, Biochem. J. 27, 1257 (1933).

[13] A. Amadi, G. Attard, J. Feliu, and A. Rodes, Langmuir 15, 2420 (1999).

[14] O. Cavalleri et al., Phys. Chem. Chem. Phys. 6, 4042 (2004).

[15] B. Hammer, L. B. Hansen, and J. K. Nørskov, Phys. Rev. B 59, 7413 (1999).

[16] M. C. Payne, M. P. Teter, D. C. Allan, T. A. Arias, and J. D. Joannopoulos, Rev. Mod. Phys. 64, 1045 (1992).

[17] D. Vanderbilt, Phys. Rev. B 41, R7892 (1990).

[18] K. Laasonen, A. Pasquarello, R. Car, C. Lee, and D. Vanderbilt, Phys. Rev. B 47, 10142 (1993).

[19] M. Mavrikakis, P. Stoltze, and J. K. Nørskov, Catal. Lett. 64, 101 (2000).

[20] S. Hüfner, J. Osterwalder, T. Greber, and L. Schlapbach, Phys. Rev. B 42, 7350 (1990).

[21] D. J. Friedman and C.S. Fadley, J. Electron Spectrosc. Relat. Phenom. 51, 689 (1990).

[22] D. Chakraborty and S. Manogaran, J. Mol. Struct. 422, 13 (1998).

[23] A complex potential energy surface with many local minima makes a search for the global minimum very difficult. Guided by experimental results, we found more favorable cysteine adsorption geometries (with up to $100 \mathrm{meV}$ lower energies) than those mentioned in Ref. [11].

[24] G. Somorjai, Top. Catal. 18, 157 (2002).

[25] R. Di Felice, A. Selloni, and E. Molinari, J. Phys. Chem. B 107, 1151 (2003).

[26] T. D. Booth, D. Wahnon, and I. W. Wainer, Chirality 9, 96 (1997). 